\title{
Scenario for Data Exchange at the Microstructure Scale
}

\author{
G . J. Schmitz ${ }^{1}$ H. Farivar ${ }^{2} \cdot$ U. Prahl ${ }^{2}$
}

Received: 4 January 2017 / Accepted: 20 January 2017 / Published online: 13 March 2017

(C) The Minerals, Metals \& Materials Society 2017

\begin{abstract}
Microstructures represent the key to interoperability between continuum models operating at the process scale and discrete models and tools describing atoms/electrons. They also provide the link between experimental materials characterisation and the virtual world. The present paper introduces a microstructure state as the central information providing the link across different length scales and along the temporal evolution of a component. Different ways of generating and representing the microstructure state are categorized and related to different classes of models acting on that state. A pragmatic way of digitally storing the microstructure state being based on the hierarchical data format HDF5 is proposed.
\end{abstract}

Keywords Digital microstructures $\cdot$ Synthetic microstructures $\cdot$ Experimental microstructures . Microstructure simulations $\cdot$ HDF5 file format . Interoperability

\section{Introduction}

Microstructures and models for their description are situated between discrete electronic, atomistic and mesoscopic models and the continuum models at the process level. Models and data explicitly or implicitly addressing microstructures, their storage and their interoperation are thus very important. The definition of a suitable scenario for a seamless information

G. J. Schmitz

g.j.schmitz@micress.de

Access e. V, Intzestr.5, 52062 Aachen, Germany

2 Steel Institute (IEHK), RWTH Aachen University, Intzestr.1, 52072 Aachen, Germany exchange between models and simulation tools drawing on microstructures from both larger and smaller scales thus is crucial. A successful scenario may be based on the description and specification of a state, which essentially corresponds to the microstructure as defined in the present document. This state can be "evolved" in time by suitable models and tools "operating" on that state. Further, this state is the carrier of the properties, which can be determined by models/tools "extracting" the necessary or desired information from that state.

The present article provides a scenario for data exchange between (i) different models/tools describing microstructures and their evolution, (ii) models/tools providing data especially for microstructure models/tools and (iii) models/tools drawing on microstructure information. The developed scenario for standardised data exchange at the microstructure level accordingly addresses the data exchange with (i) models at a larger scale, (ii) with microstructure models along the process chain and (iii) with models at smaller scales. The state description as detailed in the present article provides both a full spatial resolution character and a statistical information content, which are both necessary for interaction with models operating at the component scale and with electronic/atomistic/mesoscopic models.

\section{The Microstructure}

\section{Overview and Definitions}

The definition of "microstructures as the carrier of material properties" is widely accepted [1]. There is no process-property correlation. Instead two correlations (i) process-microstructure and (ii) microstructure-properties have to be considered. Identical processes may lead to different properties because the initial microstructure of the material was different. The first correlation 
accordingly would better read "microstructure (in)-process-microstructure (out)" [2]. Thus a proper treatment of the microstructure evolution is inevitable in ICME settings to reach a mature level of accuracy and predictive capabilities. But, how to specify and to describe microstructure information needed for different simulation models and tools? This is one of the crucial issues as it relates to the general question on how to define standards for the exchange of microstructural data. The concept of considering the microstructure as being the carrier of the properties and thus to use quantitative descriptions of the microstructure to handle the information has the advantage that real and simulated microstructures can be treated in a transparent manner.

\section{Microstructure as a System State}

According to above definitions, the microstructure defines the state of a system as it provides the basis for the description of any of its properties. The system here may be defined as a region of space being representative for the material. The state of such a system at a given instant of time is comprehensively described by a suitable set of arrays of scalars, vectors, tensors including their units.

Any evolution equation or model describing the evolution of the state of this material needs an initial state as an initial condition. Creator type models synthesize such a state by assigning values to the set of arrays of scalars, vectors, tensors including their units. Experimental microstructures can be considered as a subset of this class of creator models.

Under given boundary conditions Evolver type models will transfer the initial state into a final state after a given time period. Such models turn - under given conditions - the actual state into a new state at a later instant of time. These models accordingly change the state. They are characterized by any time dependency within the physics equation or the materials relations. Examples are the Schrödinger equation (time dependent), molecular dynamics equations, phase-field equations, diffusion equations, and Navier-Stokes equation, to name some of the most important.

Eventually, Extractor type models derive information from a given state. These models do not alter the state. These models thus "post-process" an existing state and extract desired properties from that state. Examples are mathematical homogenisation models, volume averaging, statistics tools, virtual testing and also visualisation tools like paraview (VTK).[3]

Further, different types of tools are needed to control typical ICME workflows [4]. Decisions direct the workflow e.g. based on comparing data with preset conditions and Timers/ Counters control the sequence of performing the activities and eventually input/output tools support documentation and retrieval of microstructure information.

\section{Microstructure as Carrier of Materials Properties}

Microstructures - along with the properties of the phases constituting the microstructure - are the state variables eventually determining the properties of the material. This implies that besides a thorough and comprehensive description of the microstructure state, accurate property models and equations based on the microstructure information are of equal importance. In general, only a subset of the total information provided in the microstructure state description is required - and also sufficient - to predict a specific, targeted property of a material [1]. As an example for the target property "strength" a simplified property equation can be used:

$\tau_{0}=(P)+\Delta \tau(D)+\Delta \tau(C) \Delta \tau\left(f / r_{p}\right)+.$.

with $P$ : dislocation density, D: grain size, C: solute concentration, $\mathrm{f}$ : particle volume fraction and $\mathrm{r}_{\mathrm{p}}$ : particle radius. In this example only $P, \mathrm{D}, \mathrm{C}$ and $\mathrm{f}$ are the relevant data which have to be available in the microstructure state description and then can be extracted to calculate the strength $\tau_{0}$. However, in order to derive these particular parameters $-P, \mathrm{D}, \mathrm{C}$ and $\mathrm{f}-$ from microstructure evolution simulations it is in general necessary to calculate/determine further microstructural details.

Different microstructure states will typically be present across a macroscopic component and will accordingly lead to different properties at different locations of the component. For this purpose the concept of representative volume elements (RVE) has been introduced. An RVE representation of the microstructure is characteristic and representative for a certain part of the component and the variation of the microstructure on the component level can be traced by a sufficient number of RVE's being distributed over the component. Somehow an RVE matches experiences in everyday life metallography which is usually also restricted to the important regions of a component only. The spatial distribution of the microstructure in the different RVE's over the component is the result of local variations of the process conditions like local differences in the thermal history. There is no unique microstructure for an entire component - except for single crystals or amorphous materials - and an important question relates to the number of RVE's being sufficient for a valid description of the property variation across the entire component.

\section{Types of Microstructure Data}

\section{Overview}

Material engineers aiming at developing new materials and their processing in general seek spatially resolved microstructure information as it can, for example, be obtained from micrographs and microscopy at different scales. Process engineers and component designers in contrast seek properties of materials and are 
not interested in the spatial details of the microstructure. They often draw on a number of empirical materials laws allowing estimating materials properties on the basis of statistical microstructure information. Eventually material modellers need digital, numerical representations of the microstructures.

Accordingly, the following three types of microstructure representations are important:

1. Spatial representations

2. Statistical representations

3. Numerical representations

\section{Spatial Representation of a Microstructure}

Any spatially resolved description of a microstructural state requires the use of continuum fields (scalars, vectors, tensors). Eventually, for their numerical treatment, these fields have to be discretised into data arrays discretising the system volume into numerical cells. The dimension of the arrays corresponds to the number of numerical cells in this case. A complementing approach for discrete models is the use of arrays describing e.g. the position of objects (e.g. atoms in atomistic models) with the dimension of the array corresponding to the number of objects then. Objects/features in this context are defined as regions of space revealing a contrast to their surroundings in at least one property.

Analytically, any object/feature of any size can be described by mathematical functions like e.g. feature indicator functions being defined in the continuous 3D space of real numbers [5]. Thus, space, in principle, can be discretised down to subatomic scales allowing the description of any object by continuum approaches.

Besides the feature indicator function describing shape and positions of objects/features in the microstructure, typical continuum fields in a microstructure state description are, for example, concentration fields for the individual chemical elements, stress/ strain fields, dislocation/defect densities and many others. Such fields allow accounting for variations within individual objects. A comprehensive listing of such fields is given in [5].

Spatial representations of microstructures can be classified as

- Synthetic microstructures

- Experimental microstructures

- Simulated microstructure

- Combined microstructures

\section{Synthetic Microstructures}

Synthetic microstructures may be defined as spatially resolved digital microstructures which are neither determined experimentally nor are a result of a simulation. Synthetic microstructures thus are artificially designed and created. The basis and guideline for such a design may be to match a predetermined statistical behaviour e.g. a grain size distribution or other conditions. Synthetic microstructures thus provide information being required to bidirectionally map between different representations of a microstructure. They address the "enrichment" of a statistical representation towards a spatial representation:

Statistical representation $\rightarrow$ Enrichment $\rightarrow$ Spatial representation

Spatial representation $\rightarrow$ Reduction $\rightarrow$ Statistical representation

However, current synthetic microstructures and respective tools e.g. [6-9] are still limited to the description of objects not revealing any internal structure themselves. Open questions thus relate e.g. to the synthesis of composition fields or to the synthesis of 3D structures based on 2D information. The synthesized spatial representation of the microstructure accordingly is not yet comprehensive.

\section{Experimental Microstructures}

Experimental microstructures may be defined as spatially resolved digital microstructures which are results of experimental characterisation using a variety of methods like e.g. LOM, SEM, EDX, EBSD, CT and many others.

The combination of synthetic, artificial microstructures with experimental microstructures is an important target for future developments. Currently, in many cases only 2dimensional information on microstructures is experimentally available from, for example, metallographic sections. While a $3 \mathrm{D}$ analysis is possible using serial sectioning, such an analysis is time consuming and expensive. In the future the information from two orthogonal sections might be used to derive a statistical representation of the underlying 3D microstructure. Based on this statistical representation a full 3-D spatial representation of the microstructure can then be synthesized and can be made available for numerical models/tools allowing further interpretation of experimental data and/or subsequent simulations.

A recent trend in experimental microstructure analysis is to combine and to integrate information gained from different experimental techniques providing different kinds of data, for example optical microscopy (grain sizes over large areas), EDX (element distribution on smaller scales) and EBSD (for crystallography, orientation and texture analysis).

Additional further integration of respective microstructure data with "virtual" data gained from simulations is highly beneficial for a seamless integrative concept. In the case of comprehensive microstructure simulations, similar algorithms 
for analysing experimental and virtual microstructures can be used. This will contribute to better matching models with the behaviour of the real materials and also will lead to a better understanding/interpretation of experimental results and experimental procedures.

\section{Simulated Microstructures}

The simulation of microstructures and especially of their evolution has made tremendous progress in the last decade. Phase-field, multi-phase-field, especially when being coupled to thermodynamic databases [10, 11], phase-field crystal models, crystal plasticity FEM [12] and cellular automata methods are the key ingredients of respective model chains eventually ending with failure and fatigue modelling at the end of the component life cycle e.g. $[13,14]$. A comprehensive description of respective models, methods and tools is provided in the recent Handbook of Software Solutions for ICME [15].

\section{Combined Microstructures}

The combination of different microstructures into a single description is most important, for example, for joining processes of dissimilar materials, for the development of composites, for the development of soldering and brazing materials and many others.

A standard description to communicate microstructure information based on a voxel type numerical representation seems to emerge. This present type of communication, however, does neither contain any specification for the size of the RVEs, for the size of the individual voxels, nor rules for the use of other types of grids. Procedures therefore are required in the future to adapt the RVE discretisation to the desired situation. Respective procedures concern operations on the RVE size, operations on the voxel discretisation and operations on general numerical grids.

While the formulations of the microstructure state description are basically independent of any specific numerical representation, a number of meshing functionalities still have to be developed with respect to combining microstructures being available in different numerical representations. Future actions are recommended to develop means to standardize operations on Voxel Grids (e.g. scaling voxel grids and all related information) and especially the conversion of Voxel grids to FEM grids and the conversion back from FEM grids to Voxel grids [16, 17].

\section{Statistical Representation of a Microstructure}

Current simulation and modelling tools operating at the scale of a component or describing a macroscopic process cannot digest any highly resolved microstructure information as it is provided by the full state description of a microstructure. Such models and simulation tools in general draw on statistical entities and effective values not considering any spatially resolved information. Typical values being used in these tools are, for example, average grain sizes, aspect ratios, secondary dendrite arm spacings (SDAS), grain size distributions, orientation distribution functions (ODF), phase fractions, precipitate size distributions, dislocation densities, to name only a few. The respective statistical microstructure information eventually enters into materials equations on the process scale allowing estimation of the desired properties, which might be thermomechanical properties such as the bulk modulus or the full Hook's matrix but, also functional properties like electrical conductivity or efficiency of solar cells.

It is important to note that obviously statistical values can be extracted from the spatially resolved microstructure state description as this description contains all relevant information in full (best) spatial resolution. It is less obvious — but also important to note - that statistical values can be used to synthesize a microstructure state description matching these pre-set statistics. While there are some tools already available to synthesize grain structures, there are still only few efforts to exploit detailed statistics for chemical composition of materials going beyond the average/ nominal composition of the material.

\section{Numerical Representation of a Microstructure}

To be stored in a digital manner, the spatially resolved microstructures have to be stored on a numerical grid. A priori there are no restrictions with respect to the choice of the numerical discretisation within the description of a microstructure state, for example see [5]. In practice, however, the mechanical engineering community is highly interested in FEM representations in view of their suitability for thermo-mechanical analysis. [18-19].

Materials engineers, in contrast, mostly draw on voxel type numerical representations being typical results of experimental characterisation (e.g. serial sectioning EBSD, computer tomography, serial sectioning LOM) and also of numerical simulations of microstructure evolution simulations [10] or synthetic microstructures $[7,8]$. In view of an intuitive understanding of the numerical descriptions of the microstructure state even by non-numerical experts, a voxel type representation seems to evolve as a de facto communication standard for numerical representations.

\section{Data Storage Scenario}

\section{Overview}

The microstructure state at a given instant of time is comprehensively described by a suitable set of arrays of scalars, vectors, tensors including their units. As the number of arrays and 
the variety of information they provide may become very large there is a need to provide a suitable data structure in order to make these data easily retrievable and also searchable.

Following a short specification of different data types, the need for a hierarchical data structure is highlighted and a specific implementation in an HDF5 file format is proposed. The metadata descriptors for the names of the different arrays and further metadata attributes are shortly introduced followed by a section on metadata schemata and namespaces.

\section{Data Types and Requirements}

Different communities require different types of microstructure information. While mechanical engineers working at the component scale need statistical information, materials engineers need a spatial representation. Obviously statistical values like averages, distributions, standard deviations and other types of statistical data can be extracted from the microstructure state description by a homogenisation type approach, as the state contains all relevant information in full (best) spatial resolution.

It is important to note that a statistical representation can be used to synthesize a microstructure state description matching these pre-set statistics, Figs. 1, 2 and 3.

In principle statistical representations and a spatially resolved representation thus can be mapped onto each other and can be considered as equivalent descriptions of the state. There are, however, still some limitations to be overcome, for example, with respect to synthesizing segregation patterns. Currently only the spatial representation of the microstructure state thus provides the full information depth especially with respect to chemical composition.

During the ICMEg workshops [20, 21], two major directions to describe a microstructure were identified:, either (i) staying completely on a level of statistical microstructure descriptions and just handling the parameters in the respective descriptions, or (ii) considering the microstructure as the carrier of the properties and thus to use quantitative, spatially resolved descriptions of the microstructure to handle the information.

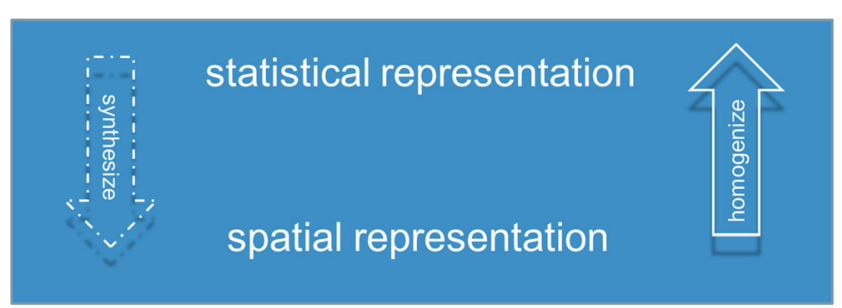

Fig. 1 Statistical representations and spatial representations of microstructures both yield valuable information. While statistical information can already be inferred from full spatial resolution data by homogenisation, a synthesis of a spatial description from statistical data still lacks statistical information especially about chemical composition (Color figure online)

\section{Specific Implementation: HDF5 Files}

All considerations above are agnostic to any specific file format. In order to achieve interoperability in practice there is, however, a need for implementing at least a file type exchange of information. The format to be selected for this purpose should be open and freely available and readable to everybody. A widespread data format, which has evolved into a communication standard already in numerous numerical communities like the CFD community, is the hierarchical data format [22]. HDF5 is a free and open source general purpose platform for storing, managing, archiving, and exchanging data. It provides extensive facilities for data and metadata association, hierarchies, and annotation along with a software library for high I/O performance, parallel I/O and out of core data access and much more.

One of the major benefits of HDF5 is its hierarchical structure matching the hierarchical nature of materials in general. Within the same data structure, HDF5 simultaneously allows for both a spatially fully resolved description and for a statistical representation of the data, making HDF5 thus very suitable to promote interoperability $[2,23]$.

\section{Metadata Descriptors}

The hierarchy of HDF5 presents as a directory-type structure which is exploitable using the free tool HDF5 view (HDF). The name(s) of the individual directory entries represent searchable keywords resp. descriptors. The specification of such metadata descriptors is a priori not an inherent part of the file structure. The specification of suitable metadata descriptors is not an easy task, since even the keywords used in some applications are "moving targets". To describe microstructures, their evolution, and the properties being associated with a given microstructure a number of different metadata descriptions are required. The metadescription of different objects within the microstructure and their mutual arrangement, i.e. the geometry of the microstructure [2], forms the backbone for many types of further attributes to these objects such as descriptors for the energetics of a microstructure or descriptors for the properties of a microstructure.

\section{Summary}

This present article paper has outlined a scenario for data exchange at the microstructure scale. This scenario is based on the definition of a state, which can be evolved and analysed by different types of models and tools.

The state essentially corresponds to a comprehensive digital description of the microstructure. It provides the initial condition for any evolution type model. The output state of 
Fig. 2 For continuum models the spatial representation essentially corresponds to fields like composition field, temperature field, feature indicator fields and others. The statistical data for continuum models are nominal composition, average grain size, phase fractions and others (Color figure online)

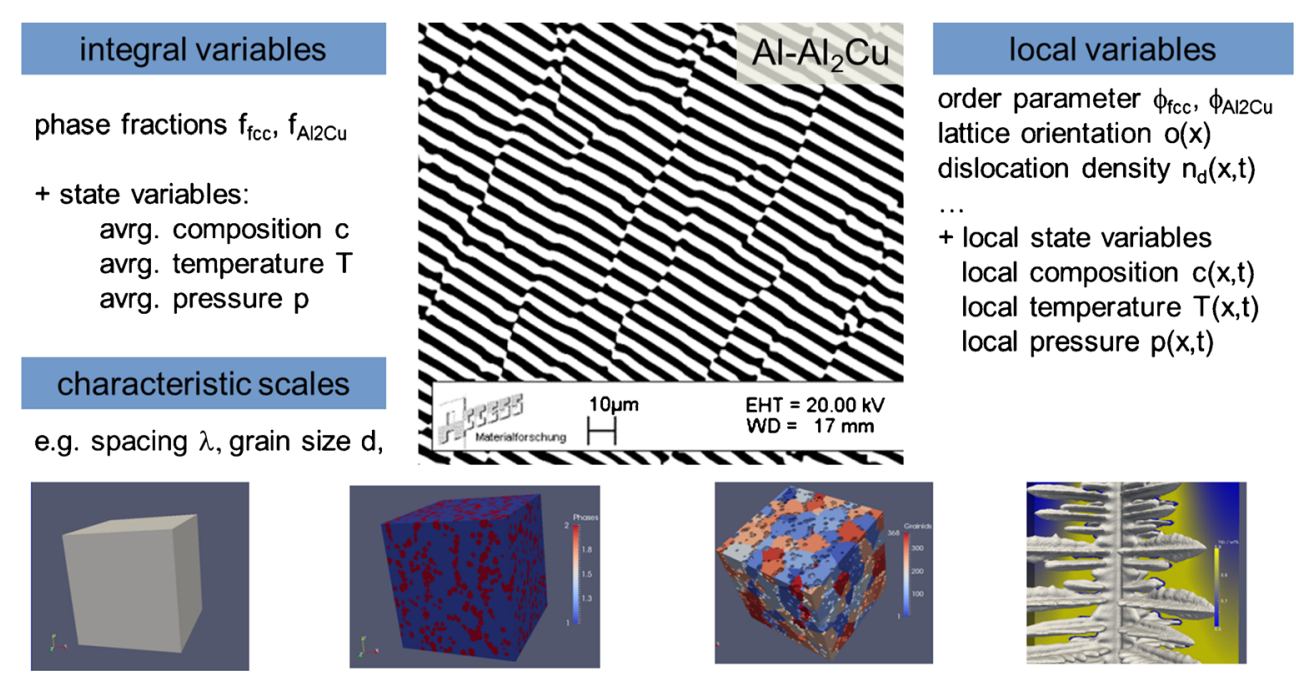

such models again represents a state, which then can be further forwarded along the simulation chain.

Models and tools have been classified into evolution type and extractor type models/tools, allowing either to evolve. to process the state corresponding to the "processing determines microstructure" paradigm, or to extract property data from the state corresponding to the "microstructure determines properties" paradigm.

The digital representation of the state is characterized by a suitable set of arrays of scalars, vectors, and tensors. A nomenclature/namespace for the metadata descriptors for each of the arrays has been proposed. This nomenclature has been elaborated on the basis of continuum models. However, it also considers descriptors for electronic, atomistic, and mesoscopic models.

A major conceptual approach is the hierarchical arrangement of the individual arrays describing the state with respect to the microstructure. This allows for different representations of the microstructure such as statistical representation and spatial representation in a coherent way within the same data structure. This inherent hierarchy of local and integral data is essential to bridge the scales between different physical phenomena and enables a seamless interaction with models/tools operating at the component scale.

The HDF5 file format [22] is proposed as a specific realisation of such a hierarchical data structure. This open source format is very powerful and versatile and already has established itself as a standard in computational fluid dynamics and in several other areas of application.

In summary, the basic concepts for interoperability between simulated, experimental and synthetic microstructures being presented in this report are now available and seem viable. These concepts are constructed so as to enable/ensure interoperability with models operating on components and processes as well as electronic/atomistic/mesoscopic models.

Future activities should relate to further spreading these concepts throughout the community, to applying them in industrial use cases, to further developing and incorporating them into workflows and simulation platforms, to broadening their scope toward uncertainty propagation and error estimates, to
Fig. 3 The spatial representation in discrete $(\mathrm{e} / \mathrm{a} / \mathrm{m})$ models corresponds e.g. to the positions and velocities of atoms of different chemical elements. Statistical data for such discrete configurations are e.g. temperature, flow velocity, or chemical composition (Color figure online)

\section{integral variables}

avrg. NumberDensity avrg. Mass/MassDensity

avrg. composition $\mathrm{c}$

avrg. vel./ temperature $\mathrm{T}$

avrg. pressure $p$

avrg. defect density

...

characteristic scales

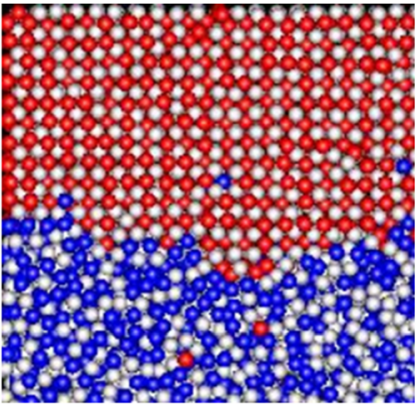

local variables

positions of atoms velocities of atoms types of atoms

... e.g. interfaces, grains, lattices, ...

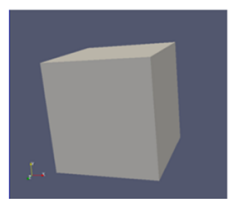

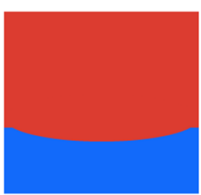

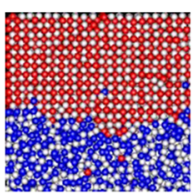


generating robust simulation chains, to harmonize namespaces and many others.

Acknowledgments The present work is based on the activities within the European Coordination and Support Action ICMEg (Grant EU FP7 6067114). It has received funding from the European Union's Horizon 2020 research and innovation programme under Grant Agreement No 723867 and from the on-going Cluster of Excellence "Integrative Production Technologies for High Wage Countries" funded by the Deutsche Forschungsgemeinschaft.

\section{References}

1. G. Gottstein: Presented at the 1st International Workshop on Software Solutions for ICME 2014; available for download from www.icmeg.info (Accessed Aug. 2016)

2. G. J. Schmitz (2016) Microstructure modeling in integrated computational materials engineering (ICME) settings: Can HDF5 provide the basis for an emerging standard for describing microstructures? JOM 68(1):77-83. doi:10.1007/s11837-015-1748-2

3. VTK-The visualisation toolkit: www.vtk.org (Accessed Jan. 2017) and paraview: free, powerful 3D visualization tool for vtk files: www.paraview.org (Accessed Jan. 2017)

4. G. J. Schmitz A flow-chart scheme for information retrieval in ICME settings. Accepted for presentation at the 4th World Congress on ICME, Ypsilanti (MI), May 2017

5. G. J. Schmitz, B. Böttger, M. Apel, J. Eiken, G. Laschet, R. Altenfeld, R. Berger, G. Boussinot, A. Viardin (2016) Towards a metadata scheme for the description of materials-the description of microstructures. Science and Technology of Advanced Materials 17(1):410-430. doi:10.1080/14686996.2016.1194166

6. Neper: Polycrystal generation and meshing. http://neper. sourceforge.net/

7. M.A. Groeber and M.A. Jackson; Integrating Materials and Manufacturing Innovation 2014, 3:5. http://www.immijournal. com/content $/ 3 / 1 / 5$
8. DREAM.3D: A Digital Representation Environment for the Analysis of Microstructure in 3D. http://bluequartz.net/ (Accessed Jan. 2017)

9. Digimat. http://www.mscsoftware.com/de/product/digimat

10. The MICRostructure Evolution Simulation Software www.micress. de. (Accessed Jan. 2017)

11. ThermoCalc. www.thermocalc.se (Accessed Jan. 2017)

12. F. Roters, C. Zhang, P. Eisenlohr, P. Shanthraj, M. Diehl: On the usage of HDF5 in the DAMASK crystal plasticity toolkit. Presented at 2nd International Workshop on Software Solutions for ICME and http://damask.mpie.de

13. Vextec. http://vextec.com/ (Accessed Jan 2017)

14. L. Adam (e-xstream), private communication

15. G.J. Schmitz, U. Prahl (eds.): Handbook of software solutions for ICME, Wiley VCH Weinheim (2016), ISBN 978-3-527-33902-0

16. L Madej, A Fular, K Banas, F Kruzel, P Cybulka and K Perzynski: Finite element discretization of digital material representation models. Presented at 2nd International Workshop on Software Solutions for ICME (http://congress.cimne.com/icme2016/frontal/ default.asp)

17. C Sandström, Chalmers University, private communication

18. A. Reid, S. Langer and S. Keshavarz: OOF: Flexible finite element modeling for materials science. Presented at 2nd International Workshop on Software Solutions for ICME

19. A.S. Bhadauria, C. Agelet de Saracibar, M. Chiumenti, M. Cervera: A new approach to interoperability using HDF5. Presented at the 2nd International Workshop on Software Solutions for Integrated Computational Materials Engineering, Barcelona, Spain, 12-15 April 2016

20. First International Workshop on Software Solutions for ICME Rolduc/Aachen, 2014 (www.icmeg.info)

21. Second International Workshop on Software Solutions for ICME Barcelona, 2016. (http://congress.cimne.com/icme2016/frontal/ default.asp)

22. The HDF group www.hdfgroup.org/HDF5/ (Accessed Jan. 2017)

23. The HDF group http://www.hdfgroup.org/products/java/hdfview/ (Accessed Jan. 2017) 